\title{
Exploiting the Potential of OLED-Based Photo-Organic Sensors for Biotechnological Applications
}

\section{Krujatz $\mathrm{F}^{1 * \#}$, Hild OR ${ }^{1 \#}$, Fehse $\mathrm{K}^{2}$, Jahnel $\mathrm{M}^{2}$, Werner $\mathrm{A}^{1}$ and Bley $\mathrm{T}^{1}$}

${ }^{1}$ Institute of Natural Materials Technology, TU Dresden, Bergstraße 120, 01069 Dresden, Germany

${ }^{2}$ Fraunhofer Institute for Organic Electronics, Electron Beam and Plasma Technology FEP, Maria-Reiche-Straße 2, 01109 Dresden, Germany

\#These authors contributed equally

\begin{abstract}
Micro- and millilitre-scale sensors have become increasingly important in modern biotechnology. Miniaturization and parallelization are also commonly employed in bio-analytical applications, environmental science and bioprocess engineering. This reduction of dimensions to the micro- and milliliter-scale stems from the demand for new sensor systems that enable non-invasive monitoring of bioprocesses in microfluidic or on-chip devices. Highly sensitive optical (bio-) sensors, with operating principles based on photoluminescence intensity or lifetime detection, hold significant promise for meeting the space-limited conditions within miniaturized biotechnological systems. In this context, the properties and applications of OLED-based organic sensors in biotechnology are discussed. The possible use of OLEDs as excitation sources in (analytical) biotechnological applications is also examined.
\end{abstract}

Keywords: Organic sensors; Photoluminescence; Microwell plates; Microfluidic devices; Photodiodes

\section{Introduction}

Miniaturized high-throughput operation systems including microwell plates [1-3], microfluidic devices [4], Lab-on-Chip (LOC) devices $[5,6]$ and low-volume bioreactors [7] have become increasingly important in modern biotechnology. These systems enable rapid, highly parallelized and automated screening and analyses of large numbers of samples. Owing to the non-invasive nature, high sensitivity and easy implementation of optical monitoring systems such as lightemitting diodes (LEDs), lasers and photodiodes are widely used as monitoring devices in these small-scale systems. As discussed in other reviews, photo-organic electronics, in contrast to aforementioned conventional optical devices, allow the design of structurally integrated miniaturized sensor arrays that provide low-cost, portable and multianalyte detection systems [8-10]. The Figure 1 shows examples of suitable orientations of integrated photo-organic sensor systems. The simplest form is associated with front-detection, where the analyte is sandwiched by the organic light emitting diode (OLED) excitation source and the photodetector [11]. However, the size of the overall device may be reduced by using a back-to-back orientation or by placing the OLED light source next to the photodetector.

\section{Classification of optical sensors used in biotechnology}

Optical sensors consisting of a light emitting device such as laser, LED, xenon or arc lamp and a photodetector (photomultiplier, photodiode) are widely used in biotechnological instruments (e.g., spectrophotometers, fluorescence microscopes or flow cytometers) and online process monitoring. Absorption, transmission and photoluminescence (PL) are the main optical effects used for analytical approaches.

Based on the measurement principle, optical sensors can be classified as PL-sensors, biosensors or absorption/transmission sensors (Figure 1). Biosensors are based on the direct coupling of a matrix-immobilized biological active receptor with a physicochemical transducer [12]. The high affinity of the receptor for a given analyte enables detection of this analyte even within complex sample structures. The receptor materials of biosensors can be distinguished among bio-catalytically active structures in enzymes, bio-affinity- active compounds, such as antibodies or nucleic acids, and whole-cell bioactive materials. PL-sensors use fluorescent dyes which are also typically matrix-immobilized, but are biologically inactive. The PL-

\section{OLED-based sensors}

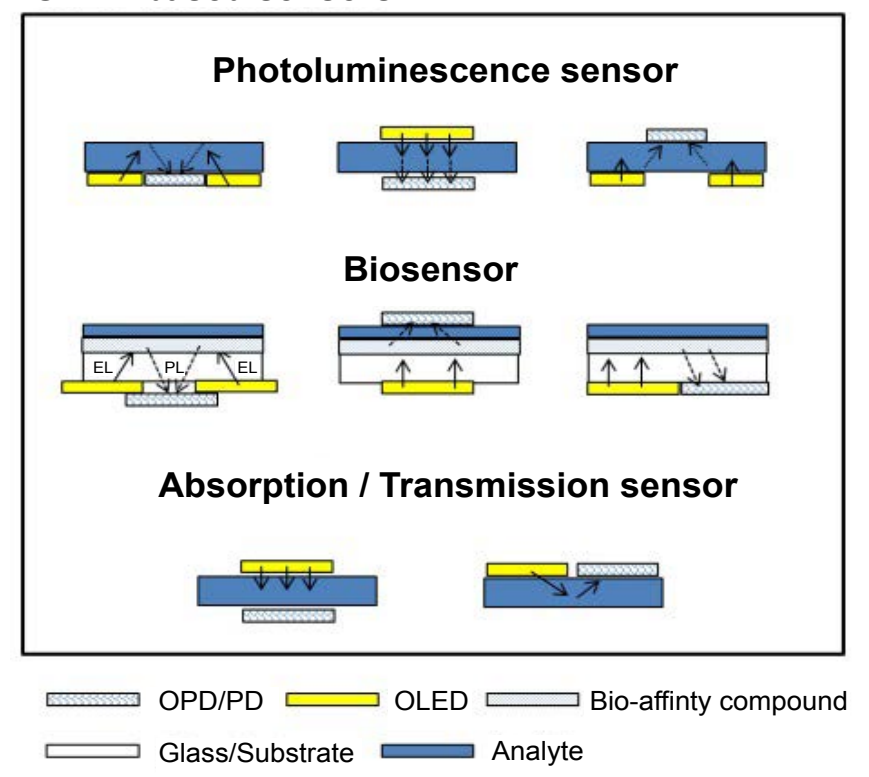

Figure 1: Orientations of OLED-based photo-organic sensor systems.

${ }^{*}$ Corresponding author: Felix Krujatz, Institute of Natural Materials Technology, TU Dresden, Bergstraße 120, 01069 Dresden, Germany, Tel: +4935146332727; Fax: +4935146337761; E-mail: Felix.Krujatz@tu-dresden.de

Received June 23, 2016; Accepted July 12, 2016; Published July 18, 2016

Citation: Krujatz F, Hild OR, Fehse K, Jahnel M, Werner A, et al. (2016) Exploiting the Potential of OLED-Based Photo-Organic Sensors for Biotechnological Applications. Chem Sci J 7: 134. doi:10.4172/2150-3494.1000134

Copyright: (C) 2016 Krujatz F, et al. This is an open-access article distributed under the terms of the Creative Commons Attribution License, which permits unrestricted use, distribution, and reproduction in any medium, provided the original author and source are credited. 
Citation: Krujatz F, Hild OR, Fehse K, Jahnel M, Werner A, et al. (2016) Exploiting the Potential of OLED-Based Photo-Organic Sensors for Biotechnological Applications. Chem Sci J 7: 134. doi:10.4172/2150-3494.1000134

properties of these dyes depend on the presence of either a specific analyte (e.g., oxygen) or the ambient conditions (e.g., pH).

Given their increasing importance, this review aims to highlight the properties and usage of OLEDs as light sources for (analytical) biotech applications. The paper consists of two main parts. In the first part, the properties of OLED-based devices are presented, with special emphasis on the state-of-the-art OLED brightness, efficiency, wavelengths and lifetimes. In the second part, recently (time frame of 10 years) developed OLED-based (bio-) sensors are reviewed and potential applications are discussed.

\section{Properties of state of the art Organic Light Emitting Diodes (OLEDs)}

OLED brightness and spectral properties: OLED technology has been valorized, i.e., progressed from research and development to commercial applications, in the last few years. OLED-based devices are used predominantly in displays for mobile applications. In fact, high-end smartphones consist mainly of active-matrix OLED displays which cost almost the same as LCD displays. HIS Technology estimates a production cost of $\$ 55$ for the Samsung Galaxy S7 5.1-inch Quad HD Super AMOLED touchscreen display [13]. However, in addition to economic factors such as costs, several technological factors (e.g., color gamut, contrast, frame rates, device thickness, complexity and power consumption) have contributed to rapid growth of the OLED market. Although OLEDs suffered from low lifetime and brightness for many years, improved materials and encapsulation technologies as well as innovative device architectures have paved the way for ongoing commercialization and strong market growth. The Figure 2 shows the basic sequence of layers in two forms of single-unit OLED devices: a bottom-emitting OLED for transparent substrates and a top-emitting OLED for opaque substrates. The transport layers enable the balance of the charge carriers inside the OLED, thereby ensuring electron-hole recombination inside the emission layer generating the light emission. The emission layer of monochromatic OLEDs is composed of a host material and an emitter which determines the color of the OLED. In addition, optical and electrical simulations of the layer sequence are extremely useful since they allow: (i) prediction of optimal layer thicknesses, for maximum light out-coupling and (ii) adjustment of the light wavelength by utilizing the micro cavity effect [14] that occurs between the anode and cathode. This is especially important in top-emitting devices where the cathode is composed of a thin semitransparent metal layer such as Ag. The device efficiency can be doubled [15] by applying additional light out-coupling measures.

In biotechnological applications, three key parameters for any OLED system used as an (excitation) light source are its brightness, spectral characteristics such as emissive light post-pulse spectrum and operational lifetime. Current commercial white-emitting OLED lighting panels [16] have a typical brightness of $3,000 \mathrm{~cd} / \mathrm{m}^{2}$ at $60 \mathrm{~lm} / \mathrm{W}$, color rendering index (CRI) of $>86$, and LT70 operational lifetimes of $\sim 10,000 \mathrm{~h}$. LT70 is defined as the lifetime corresponding to $70 \%$ retention of the initial brightness, thus after 10,000 $\mathrm{h}$ an OLED lighting device typically has a brightness of $2,100 \mathrm{~cd} / \mathrm{m}^{2}$. In fact, the OLED brightness is strongly correlated with the operational lifetime, i.e., the achievable operational lifetime decreases with increasing brightness. In the case of monochromatic OLED devices, maximum brightness values of $21,000,125,000$ and $\sim 62,000 \mathrm{~cd} / \mathrm{m}^{2}$ have been reported for blue [17], green [18] and red [19] OLEDs, respectively. The Figure 3 shows the spectral EL-properties of monochromatic OLEDs and Table 1 lists the organic emitter materials for monochromatic devices which, to date, have exhibited the highest brightness.
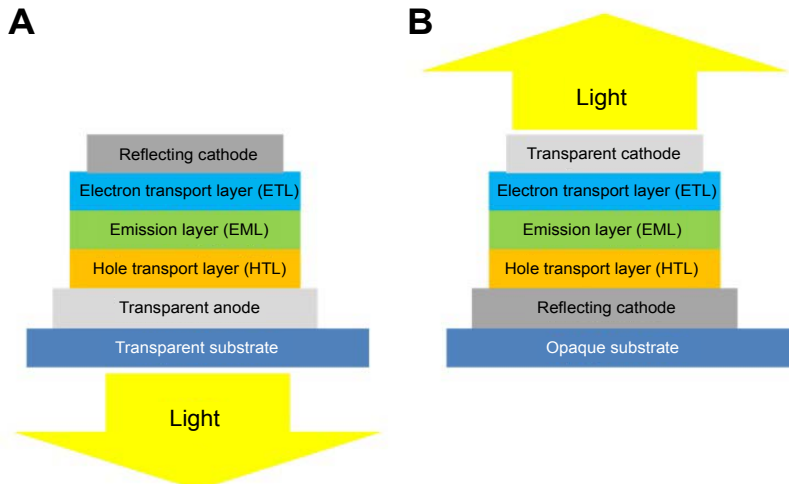

Figure 2: Layer structure of: a bottom-emitting OLED (A) and a top-emitting OLED (B).

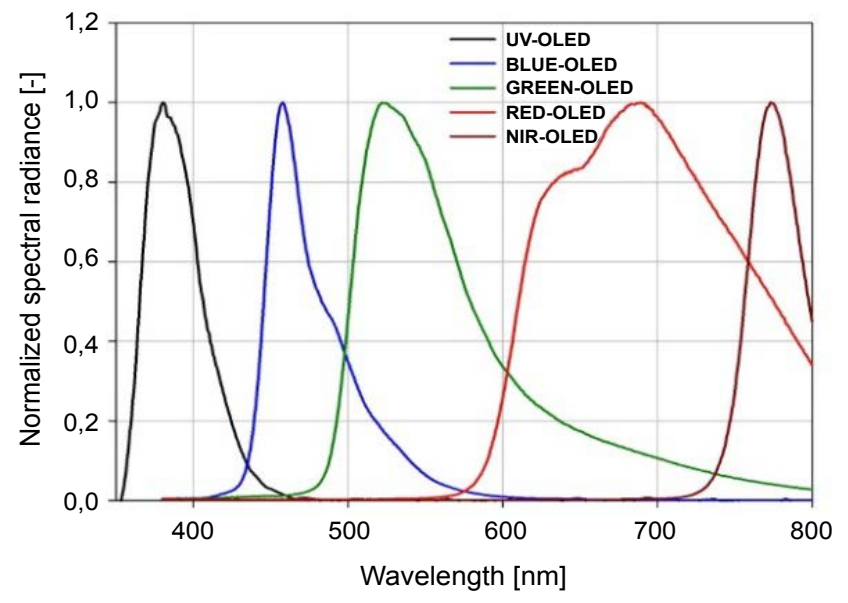

Figure 3: Normalized emission spectra of monochromatic OLEDs.

The efficiency and brightness of an OLED depend on several factors, namely the:

- Efficiency of the emitter (internal quantum efficiency)

- Matching (of HOMO and LUMO energy levels) and properties of the matrix of the emission layer (charge transfer, charge transport)

- Matching (of HOMO and LUMO energy levels) and properties of the charge transport layer HTL and ETL (charge transfer, charge transport)

- Layer thickness (optical optimization for light out-coupling and charge carrier balance)

- Conductivity, optical properties and work function of the electrodes

- Optical properties of the substrate in relation to light out-coupling

\section{Determination of OLED operational lifetimes}

The lifetime of an OLED is defined as the time-dependent decrease in the brightness at a constant current density [20-23]. This decrease results from various, internal factors [24], including: time-dependent changes in morphology, temperature effects [25] (e.g., self-heating, mechanical stress, 
Page 3 of 10

\begin{tabular}{|c|c|c|c|c|c|c|c|c|}
\hline Material & Abbreviation & Emitter type & $\lambda_{\text {em }}[\mathrm{nm}]$ & Q.Y. n (\%) & HOMO & LUMO & $\Delta \mathrm{E}$ & Source \\
\hline $\begin{array}{l}\text { 2',7'-di-tert-butyl-N,N-diphenyl-7-(4-(1-phenyl-1H- } \\
\text { benzo[d]imidazol-2-yl)phenyl)-9,9'-spirobiffluorene]- } \\
\text { 2-amine }\end{array}$ & - & blue & 457 & n.s. ${ }^{*}$ & -5.21 & -2.21 & 3.00 & 17 \\
\hline Bis(2,6-diphenylpyrimidinato) iridium (III) picolinate & $\operatorname{Ir}(\mathrm{ppm}) 2(\mathrm{pic})$ & green & 549 & 83 & -5.33 & -3.00 & 2.33 & 18 \\
\hline $\begin{array}{l}\text { Bis(2,6-diphenylpyrimidinato) iridium (III) } \\
\text { 5-(2'-pyridyl)-3-trifluromethyl-1,2,4-triazolate }\end{array}$ & $\operatorname{lr}(\mathrm{ppm}) 2(\mathrm{taz})$ & green & 523 & 86 & -5.68 & -3.26 & 2.42 & 18 \\
\hline $\begin{array}{c}\text { 4-(dicyanomethylene)- } \\
\text { 2-(1-methylcyclohexyl)-6-(1,1,7,7-tetrametyljulolidyl) } \\
\text { vinyl-4H-pyran }\end{array}$ & DCJMC & orange/red & 606 & 87 & -5.43 & -3.14 & 2.29 & 20 \\
\hline $\begin{array}{l}\text { 4-(dicyanomethylene)-2-tert-butyl-6-(1,1,7,7,- } \\
\text { tetramethyljulolidyl-9-enyl)-4H-pyran }\end{array}$ & DCJTB & orange/red & 608 & 78 & -5.26 & -3.10 & 2.16 & 20 \\
\hline $\begin{array}{c}\text { (E,E)4,7-Bis(5-(2-(9-ethylcarbazol-3-yl)ethenyl)-4- } \\
\text { hexylthien-2-yl)- } \\
\text { benzo-2,1,3-thiadiazole }\end{array}$ & - & red & 688 & n.s. & $-5,3$ & $-3,4$ & 1,9 & 21 \\
\hline Pt(II)-tetraphenyltetrabenzoporphyrin & $\mathrm{Pt}(\mathrm{tpbp})$ & NIR & 765 & n.s. & $-4,9$ & $-2,5$ & 2,4 & 22 \\
\hline
\end{tabular}

*not specified

Table 1: Overview of organic monochromatic emitter materials and the corresponding physical properties: emission wavelength ( $\lambda$ em), quantum yield, and Highest occupied molecular orbital (HOMO)/ Lowest unoccupied molecular orbital (LUMO) energy levels.

water and oxygen penetration), chemical reactions [26] (such as those induced by injected charge carriers), migration of molecules or metal clusters from electrodes and water penetration.

This lifetime, although dependent on several factors, can be predicted by an empirical scaling law [24] (equ. 1), since the OLED luminance varies with the current density over a considerable brightness range [20]. Many studies have addressed this topic [27,28]. Experimental results concur with this scaling law; $\mathrm{L}_{0}$ and $\mathrm{T}_{1 / 2}$ (also known as $\mathrm{T}_{50}$ ) are the initial luminance (i.e., luminance at time $\mathrm{t}=0 \mathrm{~h}$ ) and lifetime at $50 \%$ brightness loss compared to $\mathrm{L}_{0}$, respectively. The acceleration factor $\mathrm{n}$ is both material and device-type specific.

$$
L_{0}^{n} \times T_{1 / 2}=C
$$

Owing to the high stability of OLED devices, lifetime extrapolation based on equ. 1, yields erroneous values [29,30]. The lifetimes of devices have improved significantly in recent decades, thus present-day OLED lifetimes are typically defined as $\mathrm{T}_{70}$ or $\mathrm{T}_{90}$, corresponding to a $30-\%$ or $10-\%$ decrease compared with the initial brightness. The sharp initial decrease in brightness and slower decay after prolonged operation are governed by different degradation mechanisms. Therefore, experimental data have been fitted using equ. 2 (where $\alpha, \beta$, a and $b$ are fitting parameters) [30], which deviates from the experimental data, but is nevertheless more accurate than equ. 1 .

$$
\frac{L_{(t)}}{L_{0}}=a \times e^{-\alpha t}+b \times e^{-\beta t}
$$

To improve the fitting accuracy, alternative functions such as the stretched exponential decay (SED) [28] correlation (equation 3, where $\tau$ and $\beta$ denote the decay constant and stretching factor, respectively) have been developed to predict OLEDs' lifetime.

$$
\frac{L_{(t)}}{L_{0}}=\exp \left[-\left(\frac{t}{\tau}\right)^{\beta}\right]
$$

The lifetime of OLEDs may also be determined by fitting luminance (L) - time (t) curves, although the accuracy (of fitting) is strongly dependent on the fitted data. Further, the material type, degradation mechanisms and stack architecture all significantly affect both the lifetime and fitting accuracy.

In addition to the typical aging behavior (i.e., the time-dependent brightness decay) of OLEDs, several other visible degradation effects also occur, namely:
- Sudden degradation of luminescence: most likely due to particles or spikes/roughness of one of the electrodes, sometimes manifested as bright spots, resulting in shorting between the anode and cathode, also referred to as catastrophic failure [31].

- Shrinkage of active area and/or growth of "dark spots", nonemissive regions: This phenomenon stems mainly from water penetration of the device. OLEDs must be protected from moisture. In fact, the water vapor transmission rate (WVTR) should be lower than $10^{-6} \mathrm{~g} / \mathrm{m}^{2}$ day, i.e., $\sim 1,000000$-fold smaller than the acceptable WVTR $\left(\sim 1 \mathrm{~g} / \mathrm{m}^{2}\right.$ day $)$ for packaged food such as potato chips. In addition to water penetration through the encapsulation, water released from the substrate may also damage the OLED. The stringent moisture-protection requirements of OLEDs may be fulfilled by using encapsulation methods such as atomic layer deposition (ALD), glass frit encapsulation or plasma enhanced chemical vapor deposition (PE-CVD) [32].

OLEDs may be integrated on various surfaces, either directly, e.g., on foils or glass, or via lamination onto a specific surface. Nowadays, flexible OLEDs, which can be wrapped around, e.g., glass tubes, can be fabricated with relative ease. In 2014, Konica Minolta [33] developed white OLED lighting prototypes that exhibited a world-record efficiency of $139 \mathrm{~lm} / \mathrm{W}$. This value is significantly higher than that of the highest efficiency $(80 \mathrm{~lm} / \mathrm{W})$ [16] of current commercial OLED lighting products and fluorescent tubes, respectively. Nevertheless, it is lower than that of inorganic LEDs with efficiencies higher than $200 \mathrm{~lm} / \mathrm{W}$. However, monochromatic OLEDs with wavelengths ranging from 500-750 nm may achieve efficiencies of $>100 \mathrm{~lm} / \mathrm{W}$ [34]. Improvements in the OLED efficiency are attributed to: stacked OLED architecture, where individual OLEDs are built on top of each other; improved materials such as phosphorescent emitters; and optimization of light out-coupling.

Present-day LEDs are at least two times more efficient than OLED devices, but suffer from the drawback of being point sources and the significant effort required to generate homogenous light. In addition, LEDs generate heat locally, and thus require extensive cooling, which is highly problematic in miniaturized biotechnological applications.

\section{OLED-based (bio-) sensors in biotechnological applications}

OLED-based photoluminescence (PL) gas sensors: Organic electronics were first used in biotechnological applications as OLED- 
based devices that detect gaseous analytes. In fact, PL-sensing has been used extensively to measure amounts of gaseous or dissolved oxygen [35]. This method is based on the dynamic PL-quenching of oxygen-sensitive fluorophors such as Ru-complexes or porphyrins. The concentration of molecular oxygen directly influences the PL-intensity $(\mathrm{I})$ and PL-lifetime $(\tau)$ and can be determined from the Stern-Volmer equation which is given as:

$$
\frac{I_{0}}{I}=\frac{\tau_{0}}{\tau}=1+K_{S V}\left[O_{2}\right]
$$

where $\mathrm{I}_{0}$ and $\tau_{0}$ are the values obtained in an oxygen-free environment and $\mathrm{K}_{\mathrm{sv}}$ is the Stern-Volmer constant.

Luminescent transition metal complexes (especially $\mathrm{Ru}, \mathrm{Os}, \mathrm{Re}, \mathrm{Rh}$ and Ir complexes) have advantages for sensor applications that have been known for several decades [36]. The cited authors particularly highlighted the suitability of $\mathrm{Ru}$ (II) complexes, due to the robustness of their quenching constants. Since then, with various colleagues at Iowa State University, Ruth and Joseph Shinar extensively studied applications of OLEDs and/or organic photodetectors (OPD) for PL-based gas analysis. These studies yielded (inter alia) a structurally integrated OLED-based oxygen sensor, where excitation OLED light sources were positioned in a back-to-back orientation, with a Siphotodetector [11]. A polystyrene (PS): platinum octaethylporphyrin (PtOEP) sensor film was used as the sensing element. The back-toback-setup was subsequently used to measure the concentration of dissolved oxygen in aqueous solutions of water, ethanol and toluene [35]. In addition, the PL-intensity was enhanced by Ti-particle-doping of the PS:PtOEP sensing film, an effect attributed to light-scattering effects induced by the fine particles in the film [37].

A key challenge of PL-sensors is the spectral separation of the excitation OLED-light source, which generally exhibits a broad spectral post-pulse EL-distribution, and the PL-response signal. The sensitivity of OLED-based gas sensors was recently increased by using microcavity OLEDs ( $\mu$ c-OLEDs) to narrow the EL-spectrum. This was achieved by replacing the indium thin oxide (ITO) anode in the $\mu \mathrm{c}$-OLEDs with a semi-transparent $\mathrm{Au}$ or $\mathrm{Ag}$ thin film, leading to an increase in the EL-intensity and a decrease in the signal-to-noise-ratio (SNR). The same group subsequently replaced the PS:PtOEP sensing film with PS:polyethylene glycol (PEG) blends and combined the narrowbandwidth $\mu$ C-OLED thin-film technology with a thin-film OPD in a multicolored all-organic device. This replacement increased sensitivity by a factor of 1.9. The all-organic oxygen sensor has been further refined by using a near-UV $\mu \mathrm{C}-\mathrm{OLED}$ equipped with an Al/Pd bi-layer anode and a 4,4'-bis(9-carbazolyl)-1,1' -biphenyl emissive layer (peak emission $\approx 385 \mathrm{~nm}$ ) [38]. In contrast to the green $\mu \mathrm{C}$-OLED, the $\mu \mathrm{C}$ near UV-OLED exhibited linear-SV dependence over the entire tested range of oxygen concentration.

OLED-based PL-sensors: In the last decade, Lab-on-chip (LOC) technologies have become increasingly important in biotechnology and environmental science. The development of integrated and miniaturized sensor technologies is essential for these on-chip approaches. Photoorganic electronic devices, i.e., OLEDs in combination with OPDs, show the highest potential for fulfilling the space-limited requirements of monitoring chip-based applications since they are non-invasive, multiparametric and, generally, highly sensitive. A method for reducing the spectral crosstalk of OLED emission and the PL-signals of Rhodamine 6G (R6G) and fluorescein has been recently developed and applied in a $1-\mu \mathrm{L}$ microfluidic device with two polarizers [39]. The first polarizer was placed in front of an $\mathrm{NPB} / \mathrm{Alq}_{3}$ green-emission OLED and the other in an orthogonal orientation, thereby shielding the $\mathrm{CuPC} / \mathrm{C}_{60} \mathrm{OPD}$. This cross-polarization reduced the limit of detection (LOD) of R6G and fluorescein to $100 \mathrm{nM}$ and $10 \mu \mathrm{M}$, respectively. The LOD of both R6G and fluorescein in this type of microfluidic device was subsequently further reduced to $10 \mathrm{nM}$ [40]. A ring-shaped OPD setup for filterless distinction between excitation light and the PL-response signal has been subsequently developed in a system where OLED emission is used to excite integrated sensor spots that are sensitive to ammonia, carbon dioxide, oxygen, and temperature [41]. In an alternative, recently described strategy from our groups for controlling the light emission of OLEDs used in a R6G PL-sensor, dielectric layers, so-called distributed Bragg reflectors (DBRs), are used in narrow-bandwidth top-emitting OLEDs [42]. These DBRs are placed in front of the semi-transparent cathode. Reported results indicate that narrowing of the OLED ELemission is strongly dependent on the number of DBR pairs.

OLED-integrated dielectric mirrors may be incorporated into the architecture in several ways. The Figure 4 presents potential architectures of DBR-equipped top-emission OLEDs: a green OLED reference system fabricated on a silicon substrate (Figure 4A), an OLED device equipped with a single $\mathrm{TiO}_{2}$ layer (Figure $4 \mathrm{~B}$ ) and an OLED configuration with three DBR pairs of $\mathrm{SiO}_{2}$ and $\mathrm{TiO}_{2}$ (Figure 4C). Each system is encapsulated by a thin-film. The DBR mirror, placed on top of the OLED, functions as an optical filter, and provides maximum excitation, at a wavelength of $530 \mathrm{~nm}$, to the R6G. Moreover, the DBR has a fixed cutoff at $550 \mathrm{~nm}$, and thereby reduces the spectral overlap with the resulting R6G PL-signal. The Figure 5A exemplarily shows the OLED reference spectrum (solid green line) and its EL- (red dotted line) and PL- (blue dotted line) characteristics whereas Figure 5B shows the OLED emission spectra obtained by placing dielectric layers on the transparent cathode. The emission spectrum of the reference device (solid green line, Figure 5A) encompasses wavelengths ranging from 480 to $610 \mathrm{~nm}$, with the peak emission at $515 \mathrm{~nm}$. The Figure 5B reveals that the introduction of dielectric layers shifts the peak emission towards a wavelength of $530 \mathrm{~nm}$, and hence decreases the full width at half maximum (FWHM) value. Emission maxima of 515, 532 and $530 \mathrm{~nm}$ were obtained with $\mathrm{Al}_{2} \mathrm{O}_{3}$, a single $\mathrm{TiO}_{2}$ layer and three $\mathrm{DBR}$ pairs of dielectric layers, respectively. The peak FWHM value decreased significantly to $20 \mathrm{~nm}$ and $18 \mathrm{~nm}$ with the single $\mathrm{TiO}_{2}$ layer and DBR pairs, respectively (Figure 5B). The filter-less emission of the reference device (solid green line) exhibits significant spectral overlap at 550 $\mathrm{nm}$ with R6G fluorescence and a non-optimal peak emission for R6G excitation. In contrast to the reference, the devices equipped with a dielectric filter stimulated the pigment and yielded distinct spectral separation of OLED-EL and the R6G PL-signal. This method can be easily adapted for the design of narrow-bandwidth OLED devices operating in the ultraviolet, visible or near-infrared regions.

\section{Further potential of PL-based organic sensors}

Fluorescence dyes have been rapidly developed for various applications in biotechnology, e.g., fluorescence microscopy [43] or flow cytometry [44]. However, the use of available fluorescence dyes in organic PL-sensors remains relatively unexplored. Therefore, Table 2 provides an overview of potential biotechnological applications, based on the spectral properties of commonly used fluorescence dyes.

Therefore, the following spectral criteria, i.e., peak emission of monochromatic OLED devices, were assumed:

- UV-OLED: $385 \mathrm{~nm}$

- Blue OLED: $430 \mathrm{~nm}$

- Green OLED: $530 \mathrm{~nm}$ 
Citation: Krujatz F, Hild OR, Fehse K, Jahnel M, Werner A, et al. (2016) Exploiting the Potential of OLED-Based Photo-Organic Sensors for Biotechnological Applications. Chem Sci J 7: 134. doi:10.4172/2150-3494.1000134

Page 5 of 10

\begin{tabular}{|c|c|c|c|}
\hline \multicolumn{4}{|c|}{ UV-OLED (Peak emission: 385 nm) } \\
\hline Application & Fluorescence dye & Excitation range $[\mathrm{nm}]$ & Emission range [nm] \\
\hline \multirow[t]{8}{*}{ Imaging/Labeling } & Cascade Yellow & $360-445$ & $510-590$ \\
\hline & Marina Blue & $330-390$ & $435-495$ \\
\hline & Pacific Green & $370-450$ & $490-515$ \\
\hline & Pacific Orange & $355-435$ & $520-600$ \\
\hline & Qdot625 & $330-390$ & $610-630$ \\
\hline & Qdot800 & $300-375$ & $790-800$ \\
\hline & Dapoxyl & $325-410$ & $525-675$ \\
\hline & Dibromobimane & $340-435$ & $460-535$ \\
\hline Cell Proliferation & Cell Trace Violet Stain & $355-410$ & $435-485$ \\
\hline Nucleus Stain & DAPI & $315-390$ & $425-505$ \\
\hline $\mathrm{pH}$ Indicators & LysoSensor Yellow/Blue & $345-420$ & $505-585$ \\
\hline Cell Viability & Live/Dead Fixable Aqua Dead Cell Stain & $335-400$ & $480-575$ \\
\hline \multicolumn{4}{|c|}{ Blue OLED (Peak emission: $430 \mathrm{~nm}$ ) } \\
\hline Application & Fluorescence dye & Excitation range $[\mathrm{nm}]$ & Emission range $[\mathrm{nm}]$ \\
\hline Imaging/Labeling & PEPCP-Су5.5 & $400-550$ & $670-715$ \\
\hline \multirow[t]{3}{*}{ Nucleus Stain } & RH 795 & $435-530$ & $635-740$ \\
\hline & BCECF@pH 5.2 & $435-500$ & $500-580$ \\
\hline & LysoSensor Green & $410-485$ & $485-550$ \\
\hline \multirow[t]{2}{*}{ Cell Viability } & FM1-43 & $430-510$ & $535-710$ \\
\hline & SYPRO Protein Gel Stain & $390-500$ & $595-710$ \\
\hline $\mathrm{pH}$ Indicator & LysoSensor Yellow/Blue & $345-420$ & $505-585$ \\
\hline Viability & Live/Dead Fixable Aqua Dead Cell Stain & $335-400$ & $480-575$ \\
\hline \multicolumn{4}{|c|}{ Green OLED (Peak emission: 530 nm) } \\
\hline Application & Fluorescence dye & Excitation range $[\mathrm{nm}]$ & Emission range [nm] \\
\hline \multirow[t]{4}{*}{ Imaging/Labeling } & AlexaFluor647-R-PE & $520-580$ & $660-680$ \\
\hline & AlexaFluor680-R-PE & $520-580$ & $690-715$ \\
\hline & $\begin{array}{l}\text { Fluorospheres Nile Red (fluorescent } \\
\text { microspheres) }\end{array}$ & $480-580$ & $600-690$ \\
\hline & SNARF1@pH6 and pH9 & $480-580$ & $570-645$ \\
\hline \multirow[t]{5}{*}{ Nucleus Stain } & LDS 751 & $485-620$ & $670-765$ \\
\hline & $\begin{array}{c}\text { NeuroTrace 530/615 Red Fluorescent } \\
\text { Nissl Stain }\end{array}$ & $480-565$ & $585-685$ \\
\hline & RH 795 & $490-530$ & $690-700$ \\
\hline & Syto 80 & $470-555$ & $565-670$ \\
\hline & Syto 84 & $520-590$ & $590-690$ \\
\hline $\mathrm{pH}$ Indicator & SNARF1@pH6 and pH9 & $480-580$ & $570-645$ \\
\hline \multirow[t]{3}{*}{ Cell Viability } & 7-AAD & $490-590$ & $615-700$ \\
\hline & Ethidium Homodimer & $475-565$ & $580-685$ \\
\hline & SYTOX AADvanced Dead Cell Staining & $480-585$ & $610-700$ \\
\hline
\end{tabular}

Table 2: Overview of potential biotech applications and their associated fluorescence dyes for OLED-based photo-organic PL-sensors.

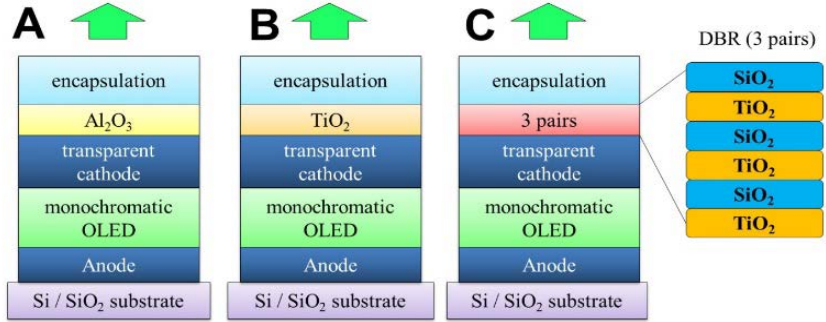

Figure 4: Schematic overview of OLED devices with integrated filter elements (A) Reference system, (B) single layer $\mathrm{TiO}_{2}$, and (C) OLED device including three DBR pairs composed of $\mathrm{TiO}_{2}$ and $\mathrm{SiO}_{2}$.

To ensure sufficient excitation of the dye and a high-intensity PLsignal, the OLED's peak EL must match sufficiently the absorption spectrum of the fluorescent dye (at least $50 \%$ ). In addition, to prevent spectral crosstalk of the OLED-EL and the PL-signal, a minimum
Stokes-shift of $75 \mathrm{~nm}$ between the EL-peak of the OLED device and the PL-peak is required (Figure 6). The research on fluorescence dye spectral properties based on the Thermo Fisher Fluorescence SpectraViewer.

\section{Transmission/Scattering measurements}

Only few applications of OLED/OPD configurations for exploiting absorption, transmission or scattering effects in biomedical applications have been reported as yet. The first example describes an OLED-integrated surface plasmon resonance device which was applied in a bio-affinity assay to quantify the interaction of a goat anti-mouse immunoglobulin $G$ and mouse IgG [45]. A second very noticeable application deals with the development of a pulse oximeter consisting of green $(E L=532 \mathrm{~nm})$ and red $(E L=626 \mathrm{~nm})$ OLEDs and OPDs fabricated on a flexible substrate [46]. When the oximeter is placed on a finger, the resulting green and red light transmission through the finger tissues provides data about the ratio of oxygenated and deoxygenated hemoglobin in the blood. This oximeter, an organic- 

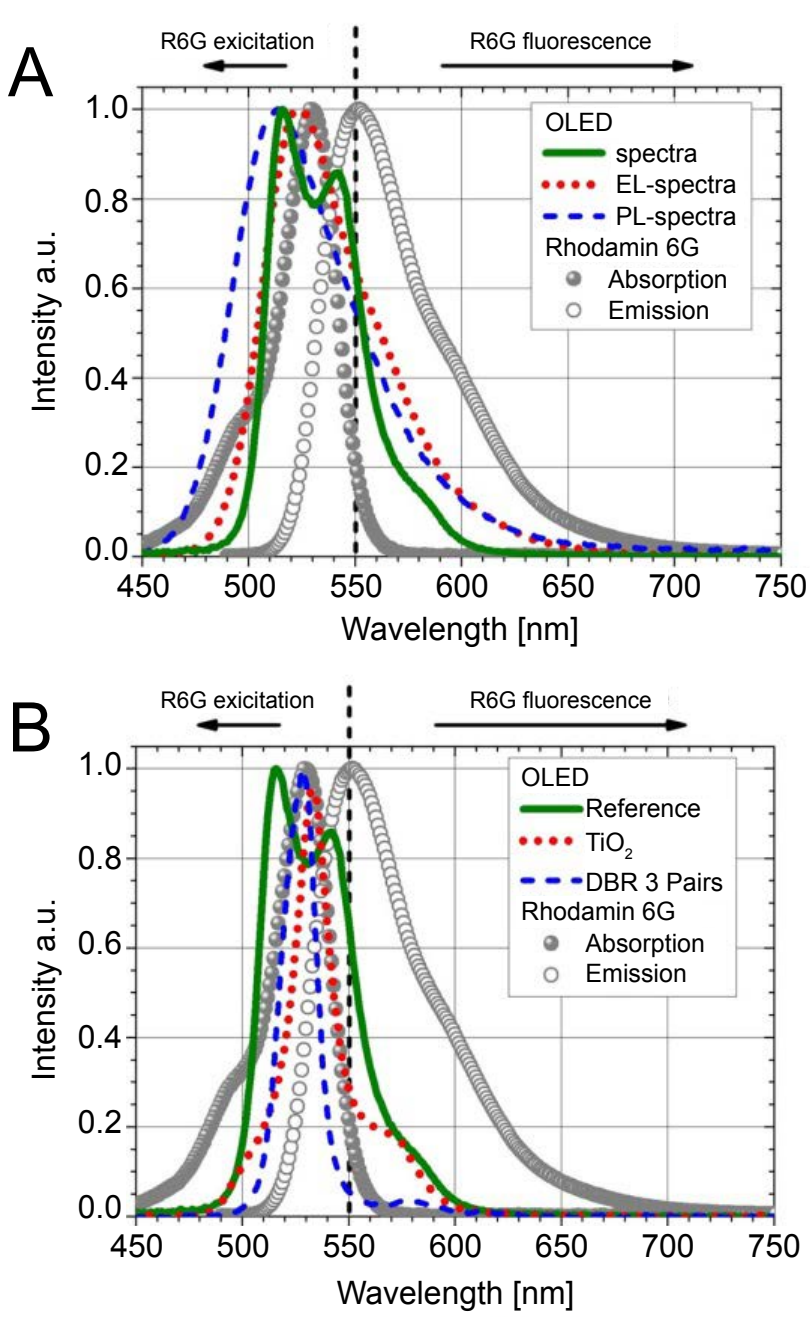

Figure 5: $(A) E L$ and $P L$ spectra of the OLED reference and $(B)$ variation in OLED emission spectra with the number of dielectric layers.

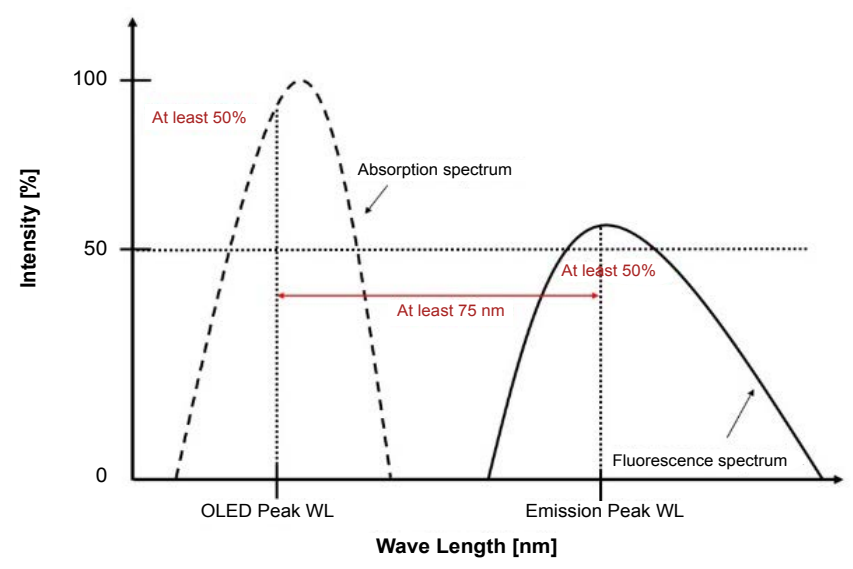

Figure 6: Spectral selection criteria for fluorescence dyes used in biotechnological applications which could be efficiently excited by monochromatic OLED-light sources. Criteria: Peak EL of an OLED device must equal at least $50 \%$ of the absorption spectrum intensity of the dye, a Stokes-shift of at least $75 \mathrm{~nm}$ between the OLED peak wavelength and the fluorescence peak wavelength and at least $50 \%$ fluorescence emission intensity are required. based device, reportedly outperforms conventional NIR-LED-based oximeters, as evidenced by error values of only $1 \%$ and $2 \%$ for pulse rate and oxygenation status, respectively.

\section{OLED-based biosensors - bio-affinity approach}

The first reported application of an OLED light source in an LOC-bio-affinity sensor involved use of a $540 \mathrm{~nm}$ bottom-emitting OLED as an excitation source, in a glass/PDMS microfluidic device, to detect Albumin Blue 580-coupled human serum albumin [47]. Moreover, instead of optical filters, the cited authors used an orthogonal detection geometry to distinguish between PL-signals and the OLED light emission. In a parallel study, a green OLED was used as an excitation source (peak emission $\approx 520 \mathrm{~nm}$ ) in a glass/PDMS microchip device [48]. An 80\% decrease in the spectral interference of the OLED light emission and the PL-response signal was achieved by placing a $300 \mu \mathrm{m}$ thin-film excitation filter on top of the OLED light source. The OLED-induced fluorescence microchip device was used for electrophoresis and fluorescence detection of R6G and Alexa 532 dyes and their bovine serum albumin-conjugates. Using the protein human tissue transglutaminase (hTG2) as a model system, a portable OLED-based protein microarray that can be used for point-of-care diagnostic applications was recently introduced [49]. Immobilized hTG 2 was printed on a cover glass and incubated first with a primary anti-hTG2 monoclonal antibody and subsequently with a secondary anti-mouse IgG antibody conjugated with AlexaFluor 430. A deep-blue a-NPB-based OLED was used to excite the AlexaFluor fluorescent dye. This resulted in specific PL-detection owing to the large $(>100 \mathrm{~nm})$ Stokes shift of AlexaFluor430 (peak excitation $=434 \mathrm{~nm}$, peak emission $=534 \mathrm{~nm}$ ). Adopting the AlexFluor430/deep-blue OLED approach for a food safety application, a miniaturized DNA-biochip was subsequently created by synthesizing a conjugate of the dye and a 55-base-long gene sequence of Campylobacter ssp [50]. Tests with real meat samples showed that the optical DNA-biochip offered 20-fold greater sensitivity than conventional plate and molecular techniques, with significant time savings.

A fluorometric flow immunoassay using an immobilized antibody for detecting alkylphenol polyethoxylates (APE) has also been recently introduced [51]. In this competitive enzyme-linked immunosorbent approach, PL-signals are collected by using a terbium complex-based green narrow-emission OLED in combination with a Fullerene 70 (C70)-based OPD. These signals are collected in two steps. In the first step, an anti-APE is immobilized in the T-shaped PDMS microfluidic channel and a mixture of APE and horse radish peroxidase (HRP)labeled APE is subsequently injected. In the second step, AmplexRed dye and $\mathrm{H}_{2} \mathrm{O}_{2}$ are injected into the channel and subsequently converted to the fluorescent product resorfin via the catalytic activity of the HRP.

A further recently reported advance in the application of OLED light sources in point-of-care diagnostics involved integration of a green OLED as an excitation source in a lateral flow immunoassay system (LFIA) [52]. The OLED was fabricated on ITO-coated PET sheets with a thin plastic excitation and emission filter covering the lateral flow test line. A quantum dot fluorophore (donkey anti-mouse antibody conjugate) is bonded to the test lines on the analytical membrane. Sensitivity studies of the quantum-dot LFIA assays, with and without OLED excitation, and conventional Au-LFIA, showed that integration of an OLED light source significantly improved the S:N ratio, signal intensity and LOD.

\section{OLED-based biosensors - bio-catalytic approach}

OLED-based bio-catalytic biosensors are structurally derived from PL-sensors that consist of a co-embedded catalytic enzymatic 
Citation: Krujatz F, Hild OR, Fehse K, Jahnel M, Werner A, et al. (2016) Exploiting the Potential of OLED-Based Photo-Organic Sensors for Biotechnological Applications. Chem Sci J 7: 134. doi:10.4172/2150-3494.1000134

Page 7 of 10

unit (Figure 7). In most applications, the substrate is converted to a product via oxygen consumption and the resulting change in oxygen concentration is revealed by PL-signals, which thus indicate the substrate consumption. A thoroughly investigated application is the enzymatic detection of glucose by immobilized glucose oxidase (GOx). For example, a back-to-back oriented OLED-based structurally integrated glucose biosensor has been developed, with either of two tested oxygen-sensitive $(\mathrm{Ru}$ or $\mathrm{Pt})$ dyes co-embedded with $\mathrm{GOx}$ in a sol-gel sensor film [53].

Excitation was achieved by using either a blue-emitting DPVBior green-emitting $\mathrm{ALq}_{3}$-OLED and the PL-intensity and lifetime were measured by a photomultiplier. The biosensor performance exhibited dependencies on both the fabrication of the sensor film and the amount of catalytically active GOx. Similar approaches for monitoring organic compounds have been developed using the catalytic activities of lactate oxidase (LOx) and alcohol oxidase (AOx) enzymes, respectively.

\section{Potential of bio-catalytic organic sensors}

There are several other potential biotechnological applications of bio-catalytic organic sensors. The Table 3 provides an overview of target molecules that could potentially be detected by bio-catalytic photo-organic sensors. Valuable targets for optical monitoring in microfluidic devices or LOC-applications include fluorescent cosubstrates such as adenosine triphosphate (ATP) [54], nicotinamide adenine dinucleotide (NADH) [55-59] and flavin adenine dinucleotide (FAD) [60]. These substances can be used to either monitor activities of various enzymes, e.g., oxygenases or dehydrogenases, which are widely applied in biotechnology.

In addition to the optical analysis of enzymatic activities via oxygen or co-substrate consumption, these substances may also be used for the direct detection of fluorescent product compounds. These include optically active pigments produced by photosynthetically active microorganisms [61], biomineralization products (such as quantum dots) [62] or water-polluting drugs (e.g., ibuprofen) [63], which could be detected by guest-host fluorescence amplification via the incorporation of cyclodextrin derivatives.

\section{OLED-based biosensors - whole-cell approach}

The first completely organic sensor device, based on cellular responses to OLED light excitation, was recently described [64]. This is

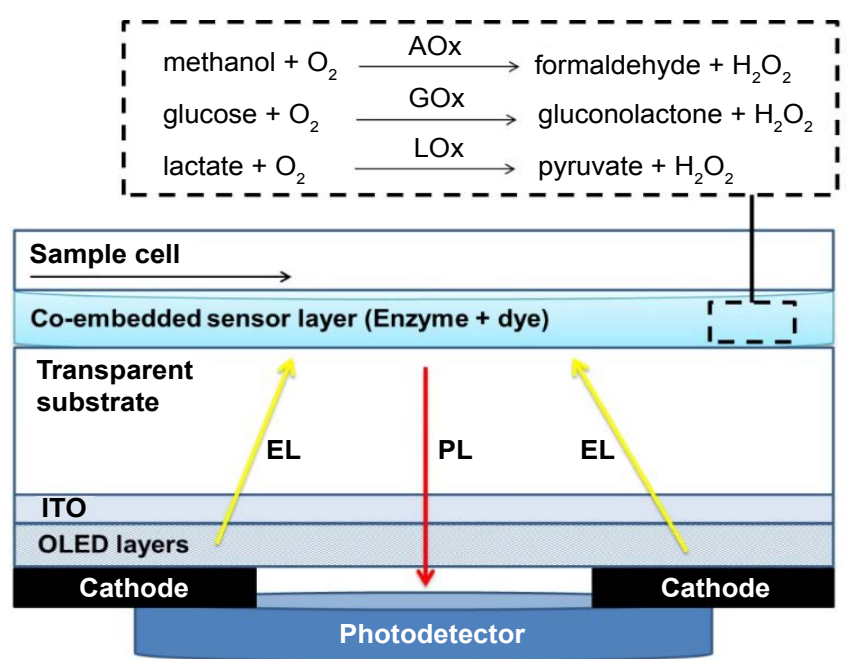

Figure 7: Illustration of the working principle of bio-catalytic-based biosensors.

\begin{tabular}{|c|c|c|c|c|}
\hline \multicolumn{5}{|c|}{ Potential for co-substrate detection } \\
\hline Co-substrate & Potential application & $\begin{array}{l}\text { Excitation } \\
{[\mathrm{nm}]}\end{array}$ & $\begin{array}{c}\text { Emission } \\
{[\mathrm{nm}]}\end{array}$ & Source \\
\hline ATP & $\begin{array}{l}\text { Monitoring of ATP-dependent } \\
\text { (bio-) reactions }\end{array}$ & 450 & 535 & 56 \\
\hline $\mathrm{NADH}$ & $\begin{array}{l}\text { Cell death detection } \\
\text { Fluorescence lifetime imaging } \\
\text { Co-enzyme regeneration } \\
\text { monitoring } \\
\text { Monitoring of various enzyme } \\
\text { activities (co-substrates) }\end{array}$ & $310-360$ & $400-550$ & $\begin{array}{l}57 \\
58 \\
59 \\
60\end{array}$ \\
\hline FAD & $\begin{array}{l}\text { Monitoring of various enzyme } \\
\text { activities (co-substrates) }\end{array}$ & $360-450$ & $500-600$ & 61 \\
\hline \multicolumn{5}{|c|}{ Fluorescent products } \\
\hline Compound & Potential applications & $\begin{array}{c}\text { Excitation } \\
{[\mathrm{nm}]}\end{array}$ & $\begin{array}{l}\text { Emission } \\
\text { [nm] }\end{array}$ & Source \\
\hline Pigments & $\begin{array}{l}\text { Astaxanthin } \\
\text { Lutein } \\
\text { ß-carotene } \\
\text { Zexanthin }\end{array}$ & $400-500$ & $>600$ & 62 \\
\hline $\begin{array}{l}\text { Quantum } \\
\text { dots }\end{array}$ & Product monitoring & $350-400$ & $400-600$ & 63 \\
\hline Drugs & $\begin{array}{l}\text { Guest-host applications } \\
\text { (cyclodextrin-drug) }\end{array}$ & $270-360$ & $280-609$ & 64 \\
\hline
\end{tabular}

Table 3: Potential applications of OLED-based bio-catalytic sensors.

a microfluidic PDMS toxicity detector, involving use of a blue OLED, $\mathrm{PTB} 3 / \mathrm{PC}_{61} \mathrm{BMOPD}$, excitation and emission filter dyes and the naturally occurring near-infrared fluorescence of the microalgal photosynthetic apparatus. In the cited study the unicellular microalga Chlamydomonas reinhardtii CC125 was exposed to varying concentrations of a herbicide (Diuron) prior to injection into the 16-channel microfluidic chip. In addition, the organic electronics, i.e., the pulsed blue OLED and OPD, and acid/base filter dyes were sandwich-oriented towards the 1-mm deep microchip channels. An $\mathrm{EC}_{50}$ of $11 \mathrm{nM}$ Diuron, for the inhibition of microalgal photochemistry, was determined using this setup. In a similar OLED-based LOC toxicity approach a portable microfluidic device consisting of three electrochemical microcells, illuminated either by a halogen light source or a blue OLED, was used [65]. After treating algal cells with selected concentrations of Diuron, the effect on the photosynthetic metabolic activity was measured via oxygen evolution and subsequent electrochemical conversion. The authors found that the sensitivity and performance of the LOC device was improved by the spectral properties of the OLED light source.

An OLED pixel-based microarray setup, consisting of $2 \times 10^{5}$ p-i-n OLED pixels deposited on a CMOS backplane, has also been introduced and applied to investigate the light-controlled phototaxis of C. reinhardtii CC125 [66].

Recently, our group developed the first OLED-based bioreactor system [67]. This device, the so-called MicrOLED-PBR, is the first FlatPanel-Airlift (FPA) photobioreactor, with a working volume of $<20 \mathrm{~mL}$ and was equipped with two white OLED modules that provide the photosynthetically active radiation for growing the microalgae strain C. reinhardtii 11-32b. OLED characteristics, such as the spectral photosynthetically active radiation efficiency, maximum photon flux density (PFD) and resulting PFD profiles across the layer thickness of the FPA-cultivation chamber, were characterized in this study.

\section{Organic (bio-) sensor array setups}

The small dimensions and potential to produce integrated photoorganic sensors can be further exploited in the fabrication of multianalyte arrays, as illustrated by the following short overview. The first was an OLED-based sensor array developed for the simultaneous 
detection of gaseous oxygen, dissolved oxygen, lactate, ethanol and glucose [68]. The back-to-back sensor platform included six pairs of green OLED pixels, each with a photodiode and an oxygen-sensitive PS:PtOEP sensor film that was used to determine the fluorescence decay time associated with oxygen quenching. Moreover, organic analytes such as glucose, lactate and ethanol were indirectly detected via the oxygen consumption catalysed by separately immobilized GOx, LOx and AOx enzymes, respectively. In another design for an all-organic multianalyte-platform a polythiophene-fullerene-based OPD was placed in front of the light source [69]. The sensor array's performance was compared when a green LED or a green OLED was used as the excitation source for simultaneous detection of glucose and oxygen. As in previous work, oxygen was monitored using the oxygen-quenching property of PtOEP, although in this case the dye was embedded in a $\mathrm{TiO}_{2}$ nanoparticle-doped PS matrix. Levels of noise at the OPD were higher when the OLED was used rather than the LED. However, this work provided proof-of-principle for such an all-organic sensor system.

The effectiveness of the filter-less ring-shaped OLED-OPD configuration discussed in the OLED-based PL-sensors section as a flexible multi-sensor chip for temperature, oxygen, $\mathrm{CO}_{2}$ and ammonia detection has been confirmed [70]. The integrated luminescent-sensor spots were therefore screen-printed on a flexible PET substrate and excited by a blue OLED. Owing to the short fluorescence lifetime (on the order of nanoseconds), the cited authors recommended use of the dual-lifetime referencing method for the $\mathrm{CO}_{2}$ and ammonia sensors.

The final system to be mentioned here is a two-parameter array equipped with spectrally-narrowed blue and green $\mu \mathrm{C}$-OLEDs as excitation sources in order to improve the forward light out-coupling efficiency and the S:N ratio [70]. The sensing matrix for monitoring oxygen was fabricated from PS:PtOEP:PEF, whereas $\mathrm{pH}$-sensitive fluorescein and a $\mathrm{CuPc} / \mathrm{C}_{70}$ OPD were used for $\mathrm{pH}$ measurements and the collection of PL-signals, respectively. Using the blue (fluorescein excitation) and green (PtOEP) $\mu$ C-OLED pixels $\left(3 \times 3 \mathrm{~mm}^{2}\right)$ as well as a high molecular-sensing matrix, yielded higher sensitivity and detectable signal intensity than those of previous multi-analyte arrays.

\section{Conclusions and Outlook}

As illustrated in this review, organic light-emitting diodes reveal numerous biotechnological applications (realized or potential) as (bio-) sensor or light source. Three features of OLED light sources are particularly important: (i) the brightness of the organic emitter, (ii) the operational lifetime and (iii) spectral characteristics of monochromatic organic emitters. The highlighted possibilities of narrowing the OLED EL-spectrum, to enable robust distinction between EL-light and PLsignals, through integrated-structural design of the light source and photodetector or the use of optical elements (filters, mirrors) are also important. The systems can be applied (inter alia) in Lab-on-Chips, microfluidic devices and cultivation systems that use OLED-based PL-sensors, biosensors, transmission sensors or photosynthetically active radiation sources for (bio-) analytical, ecological or bioprocess monitoring.

Other potential biotechnological applications of photo-organic electronics can be identified by considering the spectral properties of typically used fluorescence dyes (PL-sensors), co-substrates of enzymatic reactions (biosensors) and fluorescent substrates and products, which could be interesting targets for photo-organic monitoring. The use of organic electronics in modern small-scale biotechnological systems promises real added value since organic devices can be directly integrated. Hence, further effort is warranted to increase the flexibility of organic sensors by integrating several substrates, such as self-adhesive foils, into bioreactor systems (e.g., shake flasks, microwell systems or single-use bioreactors). The range of organo-optical sensor applications could be significantly increased by developing efficient UV-emitters since most fluorescent dyes exhibit high absorption efficiency in this spectral range. Moreover, NIR emitters should be tested in bioreactors, e.g., as monitoring devices that operate via light-scattering effects.

\section{Acknowledgements}

The authors gratefully acknowledge financial support from the Free State of Saxony (Project IDs: 4-7531.60/29/16 and 100239131).

\section{References}

1. Grünberger A, Wiechert W, Kohlheyer D (2014) Single-cell microfluidics: opportunity for bioprocess development. Curr Opin Biotech 29: 15-23.

2. Lindström S, Andersson-Svahn $\mathrm{H}$ (2011) Miniaturization of biological assays Overview on microwell devices for single-cell analyses. Biochim Biophys Acta - General Subjects 1810: 308-316.

3. Love KR, Bagh S, Choi J, Love JC (2013) Microtools for single-cell analysis in biopharmaceutical development and manufacturing. Trends Biotechnol 31: 280-286.

4. Karle M, Vashist SK, Zengerle R, von Stetten F (2016) Microfluidic solutions enabling continuous processing and monitoring of biological samples: A review. Anal Chim Acta 929: 1-22.

5. Ertl P, Sticker D, Charwat V, Kasper C, Lepperdinger G (2014) Lab-on-a-chip technologies for stem cell analysis. Trends Biotechnol 32: 245-253.

6. Jung W, Han J, Choi JW, Ahn CH (2015) Point-of-care testing (POCT) diagnostic systems using microfluidic lab-on-a-chip technologies. Microelectron Eng 132: 46-57.

7. Lattermann C, Büchs J (2015) Microscale and miniscale fermentation and screening. Curr Opin Biotech 35: 1-6.

8. Joseph S, Ruth S (2008) Organic light-emitting devices (OLEDs) and OLED-based chemical and biological sensors: an overview. J Phys D Appl Phys 41: 133001.

9. Liu R, Cai Y, Park JM, Ho KM, Shinar J, et al. (2011) Organic light-emitting diode sensing platform: Challenges and solutions. Adv Func Mater 21: 4744-4753.

10. Manna E, Xiao T, Shinar J, Shinar R (2015) Organic Photodetectors in Analytical Applications. Electronics 4: 688-722.

11. Shinar R, Ghosh D, Choudhury B, Noack M, Dalal VL, et al. (2006) Luminescence-based oxygen sensor structurally integrated with an organic light-emitting device excitation source and an amorphous Si-based photodetector. J Non-Cryst Solids 352: 1995-1998.

12. Su L, Jia W, Hou C, Lei Y (2011) Microbial biosensors: a review. Biosens Bioelectron 26: 1788-1799.

13. Andrew R, Wayne L (2016) Samsung Galaxy S7 Teardown Reveals Ho-Hum Refresh.

14. Saxena K, Jain VK, Mehta DS (2009) A review on the light extraction techniques in organic electroluminescent devices. Opt Mater 32: 221-233.

15. Gather MC, Reineke S (2015) Recent advances in light outcoupling from white organic light-emitting diodes. J Photon Energ 5.

16. LG (2016) Your Dream We Light.

17. Luo J, Zhou Y, Niu ZQ, Zhou QF, Ma Y, et al. (2007) Three-dimensional architectures for highly stable pure blue emission. J Am Chem Soc 129: 11314 11315.

18. Tong BH, Mei QB, Tian RQ, Yang M, Hua QF, et al. (2016) High-brightness solution-processed phosphorescent OLEDs with pyrimidine-based iridium(III) complexes. RSC Adv 6: 34970-34976.

19. Leung MK, Chang CC, Wu MH, Chuang KH, Lee JH, et al. (2006) 6-N $\mathrm{N}$-diphenylaminobenzofuran-derived pyran containing fluorescent dyes: a new class of high-brightness red-light-emitting dopants for OLED. Org Lett 8: 2623-2626. 
Citation: Krujatz F, Hild OR, Fehse K, Jahnel M, Werner A, et al. (2016) Exploiting the Potential of OLED-Based Photo-Organic Sensors for Biotechnological Applications. Chem Sci J 7: 134. doi:10.4172/2150-3494.1000134

20. Lee KH, Kim YK, Yoon SS, Kor B (2011) Highly Efficient Red Organic LightEmitting Diodes Using Methylcyclohexylpyran-Containing Emitters. Chem Soc 32: 4419-4422.

21. Ledwon $P$, Zassowski $P$, Jarosz T, Lapkowski M, Wagner $P$, et al. (2016) A novel donor-acceptor carbazole and benzothiadiazole material for deep red and infrared emitting applications. J Mater Chem C 4: 2219-2227.

22. Borek C, Hanson K, Djurovich PI, Thompson ME, Aznavour K, et al. (2007) Highly Efficient, Near-Infrared Electrophosphorescence from a PtMetalloporphyrin Complex. Angew Chem-Int Edit 46: 1109-1112.

23. Van Slyke SA, Chen $\mathrm{CH}$, Tang CW (1996) Organic electroluminescent devices with improved stability. Appl Phys Lett 69: 2160-2162.

24. Sato S, Ichinosawa S, Kanai H (1998) Operation characteristics and degradation of organic electroluminescent devices. IEEE J Sel Top Quant 4: 40-48.

25. Tyagi P, Srivastava R, Giri LI, Tuli S, Lee C (2016) Degradation of organic light emitting diode: Heat related issues and solutions. Synthetic Met 216: 40-50.

26. Schmidbauer S, Hohenleutner A, König B (2013) Chemical Degradation in Organic Light-Emitting Devices: Mechanisms and Implications for the Design of New Materials. Adv Mater 25: 2114-2129.

27. Aziz H, Popovic ZD (2004) Degradation phenomena in small-molecule organic light-emitting devices. Chem Mater 16: 4522-4532.

28. Scholz S, Kondakov D, Lüssem B, Leo K (2015) Degradation mechanisms and reactions in organic light-emitting devices. Chem Rev 115: 8449-8503.

29. Meerheim R, Walzer K, Pfeiffer M, Leo K (2006) Ultrastable and efficient red organic light emitting diodes with doped transport layers. Appl Phys Lett 89: 03.

30. Féry C, Racine B, Vaufrey D, Doyeux H, Cinà S (2005) Physical mechanism responsible for the stretched exponential decay behavior of aging organic lightemitting diodes. Appl Phys Lett 87: 213502.

31. Zhou X, He J, Liao LS, Lu M, Ding XM, et al. (2000) Real-time observation of temperature rise and thermal breakdown processes in organic LEDs using an IR imaging and analysis system. Adv Mater 12: 265-269.

32. Yu D, Yang YQ, Chen Z, Tao Y, Liu YF (2016) Recent progress on thinfilm encapsulation technologies for organic electronic devices. Opt Commun 362: 43-49.

33. Minolta K (2016) Green Products.

34. Seino Y, Inomata S, Sasabec H, Pu YJ, Kido J (2016) Organic Light-Emitting Devices: High-Performance Green OLEDs Using Thermally Activated Delayed Fluorescence with a Power Efficiency of over $100 \mathrm{Im} \mathrm{W-1} \mathrm{(Adv.} \mathrm{Mater.}$ 13/2016). Adv Mater 28: 2651-2651.

35. Shinar R, Zhou Z, Choudhury B, Shinar J (2006) Structurally integrated organic light emitting device-based sensors for gas phase and dissolved oxygen. Ana Chim Acta 568: 190-199.

36. Demas JN, DeGraff BA (1991) Design and applications of highly luminescent transition metal complexes. Anal Chem 63: 829A-837A.

37. Zhou Z, Shinar R, Allison AJ, Shinar J (2007) Enhanced photoluminescence of oxygen sensing films through doping with high dielectric constant particles. Adv Funct Mater 17: 3530-3537.

38. Manna E, Fungura F, Biswas R, Shinar J, Shinar R (2015) Tunable near UV microcavity OLED arrays: Characterization and analytical applications. Adv Funct Mater 25: 1226-1232.

39. Banerjee PA, Klotzkin D, Papautsky I (2008) High-sensitivity, disposable labon-a-chip with thin-film organic electronics for fluorescence detection. Lab Chip 8: $794-800$.

40. Banerjee PA, Papautsky I, Klotzkin D (2008) A polarization isolation method for high-sensitivity, low-cost on-chip fluorescence detection for microfluidic lab-ona-chip. IEEE Sens J 8: 621-627.

41. Lamprecht T, Abel E, Kraker A, Haase C, Konrad M, et al. (2010) Integrated fluorescence sensor based on ring-shaped organic photodiodes. Phys Status Solid-R 4: 157-159.

42. Jahnel M, Beyer B, Thomschke M, Fehse K, Krujatz F, et al. (2015) Narrow Bandwidth Top-Emitting OLEDs Designed for Rhodamine 6G Excitation in Biological Sensing Applications. Electronics 4: 982-994.

43. Suhling K, Hirvonen LM, Levitt JA, Chung P-H, Tregidgo C, et al. (2015) Fluorescence lifetime imaging (FLIM): Basic concepts and some recent developments. Med Photon 27: 3-40.
44. Hyka P, Lickova S, Přibyl P, Melzoch K, Kovar K (2013) Flow cytometry for the development of biotechnological processes with microalgae. Biotechnol Adv 31: 2-16.

45. Prabowo YF, Chang YY, Lee LC, Su CJ, Yu YH, et al. (2014) Application of an OLED integrated with BEF and giant birefringent optical (GBO) film in a SPR biosensor. Sensor Actuat B-Chem 198: 424-430.

46. Lochner CM, Khan Y, Pierre A, Arias AC (2014) All-organic optoelectronic sensor for pulse oximetry. Nat Commun 5.

47. Hofmann O, Wang X, deMello JC, Bradley DDC, deMello AJ (2005) Thin-film organic photodiodes as integrated detectors for microscale chemiluminescence assays. Lab Chip 5: 863-868

48. Yao G, Luo L, Wang Y, Gao G, Lei K, et al. (2005) A microfluidic device using a green organic light emitting diode as an integrated excitation source. Lab Chip 5: 1041-1047.

49. Marcello D, Sblattero C, Cioarec C, Maiuri P, Melpignano P (2013) A deep-blue OLED-based biochip for protein microarray fluorescence detection. Biosens Bioelectron 46: 44-47.

50. Manzano M, Cecchini F, Fontanot M, lacumin L, Comi G, et al. (2015) OLEDbased DNA biochip for Campylobacter spp. detection in poultry meat samples. Biosens Bioelectron 66: 271-276.

51. Liu R, Ishimatsu R, Yahiro M, Adachi C, Nakano K, et al. (2015) Fluorometric flow-immunoassay for alkylphenol polyethoxylates on a microchip containing a fluorescence detector comprised of an organic light emitting diode and an. Talanta 134: 37-47.

52. Venkatraman V, Steckl AJ (2015) Integrated OLED as excitation light source in fluorescent lateral flow immunoassays. Biosens Bioelectron 74: 150-155.

53. Choudhury, Shinar R, Shinar J (2004) Glucose biosensors based on organic light-emitting devices structurally integrated with a luminescent sensing element. J Appl Phys 96: 2949-2954.

54. Moro J, Cywinski PJ, Korsten S, Mohr GJ (2010) An ATP fluorescent chemosensor based on a $\mathrm{Zn}$ (II)-complexed dipicolylamine receptor coupled with a naphthalimide chromophore. Chem Commun 46: 1085-1087.

55. Hsing-Wen W, Yau-Huei W, Han-Wen G (2009) Reduced Nicotinamide Adenine Dinucleotide (NADH) Fluorescence for the Detection of Cell Death. Anti-Cancer Agent Me 9: 1012-1017.

56. Blacker TS, Mann ZF, Gale JE, Ziegler M, Bain AJ, et al. (2014) Separating $\mathrm{NADH}$ and NADPH fluorescence in live cells and tissues using FLIM. Na Commun 5.

57. Quinto T, Köhler V, Ward TR (2014) Recent trends in biomimetic NADH regeneration. Top Catal 57: 321-331.

58. Barry SM, Challis GL (2013) Mechanism and catalytic diversity of rieske nonheme iron-dependent oxygenases. ACS Catal 3: 2362-2370.

59. Islam M, Honma M, Nakabayashi T, Kinjo M, Ohta N (2013) pH Dependence of the Fluorescence Lifetime of FAD in Solution and in Cells. Int J Mol Sci 14: 1952-1963.

60. Tokarz R, Cisek M, Garbaczewska D, SandkuijI X, Qiu B, et al. (2012) Carotenoid based bio-compatible labels for third harmonic generation microscopy. Phys Chem Chem Phys 14: 10653-10661.

61. Dunleavy R, Lu L, Kiely CJ, McIntosh S, Berger BW (2016) Single-enzyme biomineralization of cadmium sulfide nanocrystals with controlled optical properties. P Nat Acad Sci 113: 5275-5280.

62. Elbashir NFA, Dsugi TO, Mohmed M, Aboul-Enein HY (2014) Spectrofluorometric analytical applications of cyclodextrins. Luminescence 29: 1-7.

63. Lefevre A, Chalifour L, Yu V, Juneau CP, Izquierdo R (2012) Algal fluorescence sensor integrated into a microfluidic chip for water pollutant detection. Lab Chip 12: $787-793$

64. Tsopela A, Laborde L, Salvagnac V, Ventalon E, Bedel-Pereira I, et al. (2016) Development of a lab-on-chip electrochemical biosensor for water quality analysis based on microalgal photosynthesis. Biosens. Bioelectron 79: 568-573.

65. Steude M, Jahnel M, Thomschke M, Schober, Gather MC (2015) Controlling the behavior of single live cells with high density arrays of microscopic OLEDs. Adv Mater 27: 7657-7661.

66. Krujatz K, Fehse M, Jahnel C, Gommel C, Schurig F, et al. (2016) MicrOLED photobioreactor: Design and characterization of a milliliter-scale Flat-PanelAirlift-photobioreactor with optical process monitoring. Algal Res 18: 225-234. 
Citation: Krujatz F, Hild OR, Fehse K, Jahnel M, Werner A, et al. (2016) Exploiting the Potential of OLED-Based Photo-Organic Sensors for Biotechnological Applications. Chem Sci J 7: 134. doi:10.4172/2150-3494.1000134

67. Cai Y, Shinar R, Zhou Z, Shinar J (2008) Multianalyte sensor array based on an organic light emitting diode platform. Sensor Actuat B-Chem 134: 727-735.

68. Nalwa KS, Cai Y, Thoeming AL, Shinar J, Shinar R, et al. (2010) Polythiophenefullerene based photodetectors: Tuning of spectral response and application in photoluminescence based (bio) chemical sensors. Adv Mater 22: 4157-4161.
69. Jakoby MJ, Vellekoop T, Mayr T, Abel E, Kraker S, et al. (2010) An optical sensor array on a flexible substrate with integrated organic opto-electric devices. Procedia Eng 5: 1005-1008.

70. Liu R, Xiao T, Cui X, Shinar J, Shinar R (2013) Multiple approaches for enhancing all-organic electronics photoluminescent sensors: Simultaneous oxygen and pH monitoring. Anal Chim Acta 778: 70-78. 